\title{
Ependymomas of the spinal cord and cauda equina: An analysis of 26 cases and a review of the literature
}

\author{
T Asazuma*,1, Y Toyama ${ }^{2}$, N Suzuki $^{3}$, Y Fujimura $^{2}$ and K Hirabayshi ${ }^{3}$ \\ ${ }^{1}$ Department of Orthopaedic Surgery, National Defense Medical College, Tokorozawa, Japan; ${ }^{2}$ Department of \\ Orthopaedic Surgery, Keio University, School of Medicine, 35 Shinanomachi, Shinjuku-ku, Tokyo 160, Japan; ${ }^{3}$ Keio \\ Junior College of Nursing, 35 Shinanomachi, Shinjuku-ku, Tokyo 160, Japan
}

\begin{abstract}
Study design: Retrospective review.
Objectives: To clarify the clinical features of patients with spinal ependymomas and to compare the clinical results between the patients in whom microsurgical technique and spinal cord monitoring were used intraoperatively and the patients in whom they were not used.

Setting: Keio University Hospital, Tokyo, Japan.

Methods: Twenty-six consecutive patients with spinal ependymomas were treated surgically between 1958 and 1995. All patients underwent tumor resection through a posterior approach. Complete tumor resection was possible in 15 patients $(57.7 \%)$, and subtotal tumor resection (more than $90 \%$ ) was done in two patients $(7.7 \%$ ). Only a partial tumor resection (less than $90 \%$ ) was performed in the remainder of the patients $(34.6 \%)$. The operative results of the patients were evaluated by the Japanese Orthopaedic Association Scoring System (JOA score) and its recovery rate.

Results: The overall average recovery rate was $18.3 \%$. The mean recovery rate was $14.4 \%$ in cervical lesion, $11.1 \%$ in thoracic lesion and $40 \%$ in lumbar lesion. The recovery rate of eight patients with cervical ependymomas who underwent tumor resection under both microscopic surgical procedure and intraoperative spinal cord monitoring was $37.1 \%$ although the recovery rate of the rest of the patients was $-1.6 \%$. There was a statistical difference between the two groups $(P<0.02)$. The survival rate of patients following complete excision was statistically better compared to that of patients after incomplete resection.

Conclusion: Both microsurgical technique and spinal cord monitoring are indispensable to achieve total removal of ependymomas and to obtain improvement of neurological recovery.
\end{abstract}

Keywords: ependymoma; spinal cord; microsurgical technique; spinal cord monitoring

\section{Introduction}

Intraspinal ependymomas account for five to $52.5 \%$ of ependymomas of the central nervous system. ${ }^{1-3}$ Although a large number of spinal cord and cauda equina ependymomas have been reported, most of the published literature centers around ependymomas of the intracranial region. In recent years, the diagnosis of spinal cord tumors has improved with the advent of magnetic resonance imaging (MRI). The clinical results of surgical treatment of spinal ependymomas have been improving as a result of the use of microsurgical techniques and spinal cord monitoring intraoperatively. ${ }^{4-8}$ However, few published reports have compared the difference in clinical results between patients in whom microsurgical techniques and spinal cord monitoring were used intraoperatively and

*Correspondence: T Asazuma, Department of Orthopaedic Surgery, National Defense Medical College, 3-2, Namiki, Tokorozawa 359, Japan patients in whom they were not used. We analyzed the clinical features of patients with spinal ependymomas, and also compared outcomes between patients in whom microsurgical techniques and spinal cord monitoring were used intraoperatively and patients in whom they were not used.

\section{Materials and methods}

Between 1958 and 1995, 26 patients (13 males and 13 females) with spinal ependymomas received surgical treatment in the Department of Keio University Hospital and affiliated institutions. All the patients had histologically proven spinal cord ependymomas. The ages ranged from $11-65$ years (mean 42.6 years). Fifteen of the patients $(57.7 \%)$ were in their fourth and fifth decades. The follow-up periods ranged from 1-22 years (mean, 6 years and 1 month). MRI was used in 16 patients preoperatively, with the other ten patients 
undergoing either a myelogram and/or CT-myelogram. In six of these patients $(23.1 \%)$ the tumor was in the cervical region, in five $(19.2 \%)$ it was in the cervicothoracic region, in four $(15.4 \%)$ it was in the thoracolumbar region and in three $(11.5 \%)$ it was in the thoracic region. The number of affected spinal segments ranged from one to 24 (mean, 6.2) (Figure 1). The most common presenting symptoms were low back pain $(38.5 \%)$, back pain and neck pain $(15.4 \%)$, dysuria, numbness of the hands, leg pain, and lower extremity weakness $(7.7 \%)$. Duration between the onset of initial symptoms and the first operation ranged from 3 months to 10 years (mean, 3 years and 4 months).

Intervals between onset of symptoms and the first operation and the numbers of affected spinal segments were compared between patients treated before and after the advent of MRI to evaluate the diagnostic usefulness of such imaging. The operative results of these patients were evaluated by the Japanese Orthopaedic Association Scoring System (JOA score). This score has 17 points in all, consisting of 4 points for motor dysfunction of the upper and lower extremities, respectively; 2 points for sensory dysfunction of the upper and lower extremities and trunk, respectively; and 3 points for bladder dysfunction (Table 1). A total of 17 points could be accumulated for a cervical lesion, 11 points for a thoracic lesion (eliminating 4 points for upper extremity motor dysfunction and two points for sensory dysfunction of the upper extremities) and 9 points for a lumbar lesion (eliminating 4 points for upper extremity motor dysfunction and 4 points for sensory dysfunction of the upper extremities and trunk). The recovery rate was calculated by Hirabayashi's method. Less than $-10 \%$ was defined as worsening, more than $10 \%$ was defined as improved and between $-10 \%$ and $10 \%$ was defined as unchanged. Fourteen patients had cervical lesion (17 points full mark), six had a thoracic lesion (11 points full mark) and six had a lumbar lesion (9 points full mark). The survival rate was calculated by Kaplan-Meier's method.

\section{Treatment}

All patients underwent tumor resection through a posterior approach. A laminectomy was performed in 19 patients $(73.1 \%)$, bilateral open door laminoplasty in six patients $(23.1 \%)$ and a hemilaminectomy in one patient $(3.8 \%)$. Complete tumor resection was possible in 15 patients $(57.7 \%)$, and subtotal tumor resection (more than $90 \%$ ) was done in two patients $(7.7 \%$ ). Only a partial tumor resection (less than 90\%) was performed in the remainder of the patients $(34.6 \%)$. Shunt surgery was performed in two patients. In the last few years, microscopic surgery was done in 15 patients, with sensory and motor-evoked potentials used in nine patients intraoperatively. Spinal cord monitoring was performed in the patients with cervical or thoracic spinal cord tumors. We routinely stopped
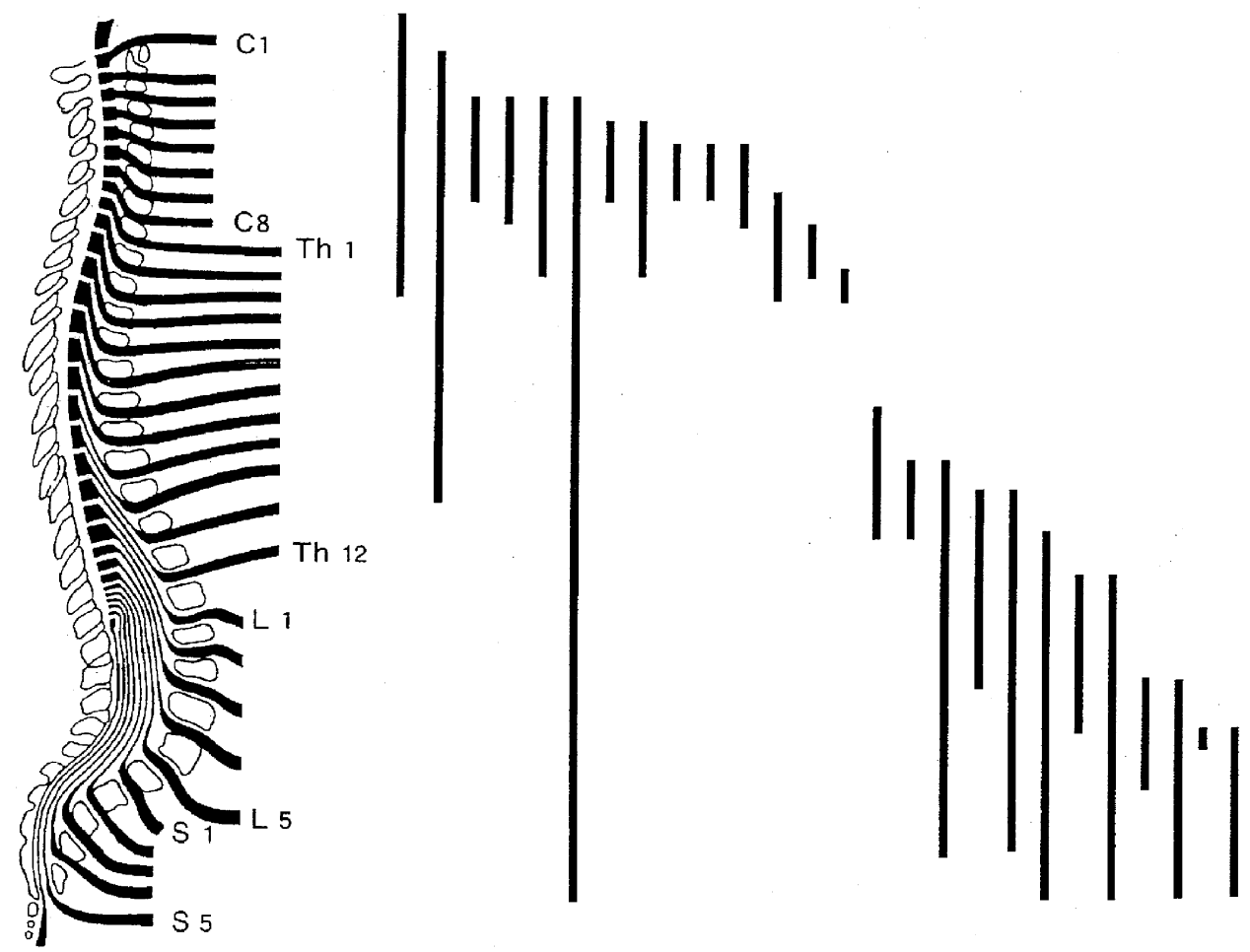

Figure 1 The distribution of the affected spinal segments. The lines stand for the distribution of the affected spinal segments 
Table 1 Scoring system for cervical myelopathy (Japanese Orthopaedic Association)

(A) Motor function

I. Upper extremity function

0 : Unable to feed oneself with any tablewares including chopsticks, a spoon or fork

1: Can manage to feed oneself with a spoon and/or fork but not with chopsticks

2: Chopstick-feeding is possible but not practical

3: Chopstick-feeding is clumsy but practical

4: Normal

II. Lower extremity function

0 : Unable to walk by any means

1: Unable to walk without a cane or other support on a level

2: Walks independently on a level but needs support on stairs

3: Capable of fast walking but clumsy

4: Normal

(B) Sensory function

I. Upper extremity

0: Apparent sensory loss

1: Minimal sensory loss

2: Normal

II. Lower extremity

0: Apparent sensory loss

1: Minimal sensory loss

2: Normal

III. Trunk

0: Apparent sensory loss

1: Minimal sensory loss

2: Normal

(C) Bladder function

0: Urinary retention and/or incontinence

1: Sense of retention and/or dribbling and/or thin stream and/or incomplete continence

2: Urinary retardation and/or pollakiuria

3: Normal

Recovery rate of JOA score

postoperative score - preoperative score

$\begin{gathered}\text { Recovery rate } \\ (\%)\end{gathered} \quad \times 100$

the surgical procedures if the amplitude of the wave decreased to less than $50 \%$. The surgery was performed only once in 19 patients, twice in three patients and three times in four patients. The interval between the first and the second procedure ranged from 6 months to 27 years (mean, 5 years and 3 months) and the interval between the second and the third procedure ranged from 1 year and 9 months to 19 years (mean, 5 years and 1 month).

Postoperative radiotherapy was used in ten patients who had partial or subtotal tumor resection. Only one patient received postoperative chemotherapy.

\section{Results}

The average duration between the onset of initial symptoms and the first operation was 3 years and 10 months (range of $1-10$ years) before the routine use of MRI, and it was 3 years and 1 month (range 5 months to 9 years) after the use of MRI. There was no statistical difference between the two groups. The number of affected spinal segments ranged from $4-$ 24 segments (mean, 8.0) before the MRI was introduced, and from 1-17 segments (mean, 4.8) after the use of MRI. There was no statistical difference between the two groups.

Thirteen patients $(50 \%)$ showed clinical improvement and seven patients $(26.9 \%)$ were unchanged, while six patients $(23.1 \%)$ demonstrated neurologic deterioration. Among cervical lesions, ten patients (71.4\%) improved, one patient $(7.1 \%)$ was unchanged, and three patients $(21.4 \%)$ deteriorated. Among thoracic lesions, one patient $(16.7 \%)$ improved, two patients $(33.3 \%)$ were unchanged, and three patients $(50 \%)$ deteriorated. Among lumbar lesions, two patients $(33.3 \%)$ improved and four patients $(66.7 \%)$ were unchanged (Table 2).

The overall average recovery rate was $18.3 \%$, ranging from $-100 \%$ to $100 \%$. The recovery rate ranged from $-100 \%$ to $69.2 \%$ (mean $14.4 \%$ ) for the cervical lesions, $-16.7 \%$ to $50 \%$ (mean, $11.1 \%$ ) for the thoracic lesions, and $0 \%$ to $100 \%$ (mean, $40 \%$ ) for the lumbar lesions. The recovery rate of the eight patients with cervical ependymomas who underwent tumor resection utilizing both the microscopic surgical procedures and intraoperative spinal cord monitoring was $37.1 \%$, but the recovery rate of the rest of the patients was $-1.6 \%$. There was a statistical difference between the two groups $(P<0.02)$. All the patients who underwent cervical spinal cord tumor resection utilizing the microscopic surgical procedures and intraoperative spinal cord monitoring improved neurologically, whereas $50 \%$ of the rest of the patients (three of six patients) deteriorated.

The differences in surgical outcomes between the 15 patients with cervical or thoracic spinal cord tumor treated microsurgically with spinal cord monitoring and without monitoring were examined. The recovery rate of the nine patients treated microsurgically with spinal cord monitoring was $41.9 \%$, but the recovery rate of six patients treated microsurgically without monitoring was $0.5 \%$. There was a statistical difference between the two groups $(P<0.02)$.

The overall mortality was $23.1 \%$ (six patients). All six underwent surgical treatment prior to 1985 . Two

Table 2 Clinical results of spinal ependymomas (No. of patients)

\begin{tabular}{lccc}
\hline & Improved & Unchanged & Deteriorated \\
\hline Cervical & 10 & 1 & 3 \\
Thoracic & 1 & 2 & 3 \\
Lumbar & 2 & 4 & 0 \\
\hline
\end{tabular}




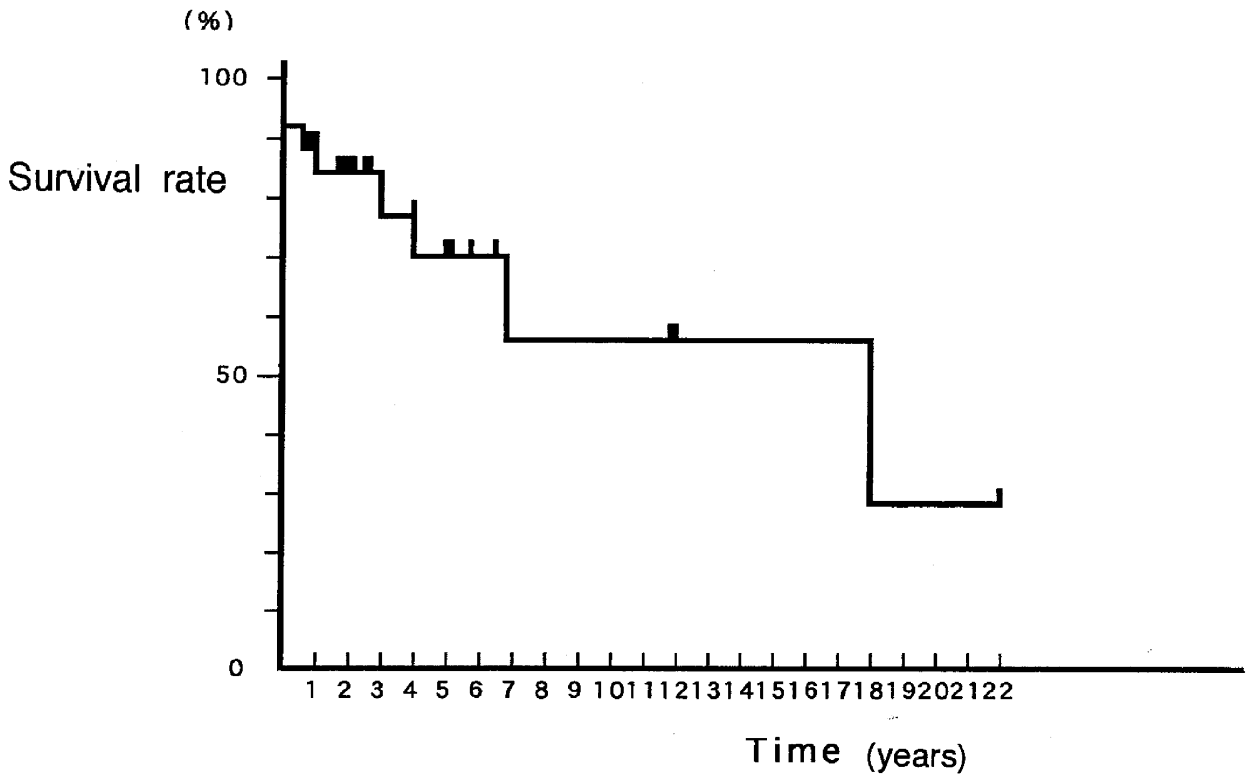

Figure 2 The overall survival rate of the spinal ependymomas

patients died of respiratory failure, one died of sepsis, and three died of unknown causes. The tumors were only partially resected in all of the mortality cases. The survival rate was as follows: $84 \%$ at 1 year, $77 \%$ at 3 years, and $70 \%$ at 5 years (Figure 2). With regard to the surgical treatment, all the patients who underwent total resection were living at 5 years, while the survival rate was $67.5 \%$ at 1 year, $56.2 \%$ at 3 years, and $45 \%$ at 5 years in patients treated with partial or subtotal resection. This difference was statistically significant $(P<0.05)$. Based on the affected segments, the survival rate was $95 \%$ at 1 year, $85.5 \%$ at 3 years and $76 \%$ at 5 years in patients with less than nine segments, and $50 \%$ at 3 years and $50 \%$ at 5 years in those with more than ten segments. There was no statistical difference between the groups. None of the patients who underwent complete tumor resection had a clinical recurrence, whereas eight of those who underwent partial or subtotal resection developed a recurrence.

\section{Case report}

A 43-year-old woman, first noticed numbness on her right fingers in May 1992. She was evaluated at our clinic in May 1993. Her preoperative JOA score was 10. An intramedullary spinal cord tumor was seen at the second cervical level extending to the first thoracic level on MRI (Figure 3). Microscopic total resection of the tumor was performed using a bilateral opendoor laminoplasty with intraoperative spinal cord monitoring (Figure 4). Her postoperative JOA score improved to 13 points, and the improvement rate was $43 \%$.

\section{Discussion}

Numerous reports on the clinical and radiologic features, the usefulness of postoperative radiation, and long term prognosis of ependymomas have been published. ${ }^{3,9-12}$ The most common presenting symptoms were reported to be back pain by Barone et $a l^{9}$ and back/neck pain by Rawlings et al, ${ }^{13}$ although the symptoms varied. It appears that pain is the most common presenting complaint in adult patients with spinal cord tumors. However, indirect phenomena such as peritumoral edema, cyst formation, and vascular derangements can produce clinical effects at a considerable distance from the tumor. ${ }^{8}$ In our study, the duration between the onset of initial symptoms and the first operation was long (mean, 3 years and 4 months), with little change after MRI was introduced (mean, 3 years and 1 month). According to the literature, the average duration of symptoms preceding diagnosis was 13 months to 8.3 years. ${ }^{7,8,12}$ Despite the sensitivity of MRI, preoperative symptom duration remains characteristically long. 8

The overall 5-year survival rate of the spinal ependymomas was reported to be between 57 and $90 \% .^{3,11,12,14}$ The overall 5-year survival rate was $70 \%$ in our study, it was similar to the previous reports. The survival rate of patients following complete excision was statistically better compared to that of patients after incomplete resection in our study as Whitaker et al ${ }^{11}$ demonstrated. However, long term follow-up is needed to definitively conclude the survival rate of patients treated with complete or incomplete resection. 

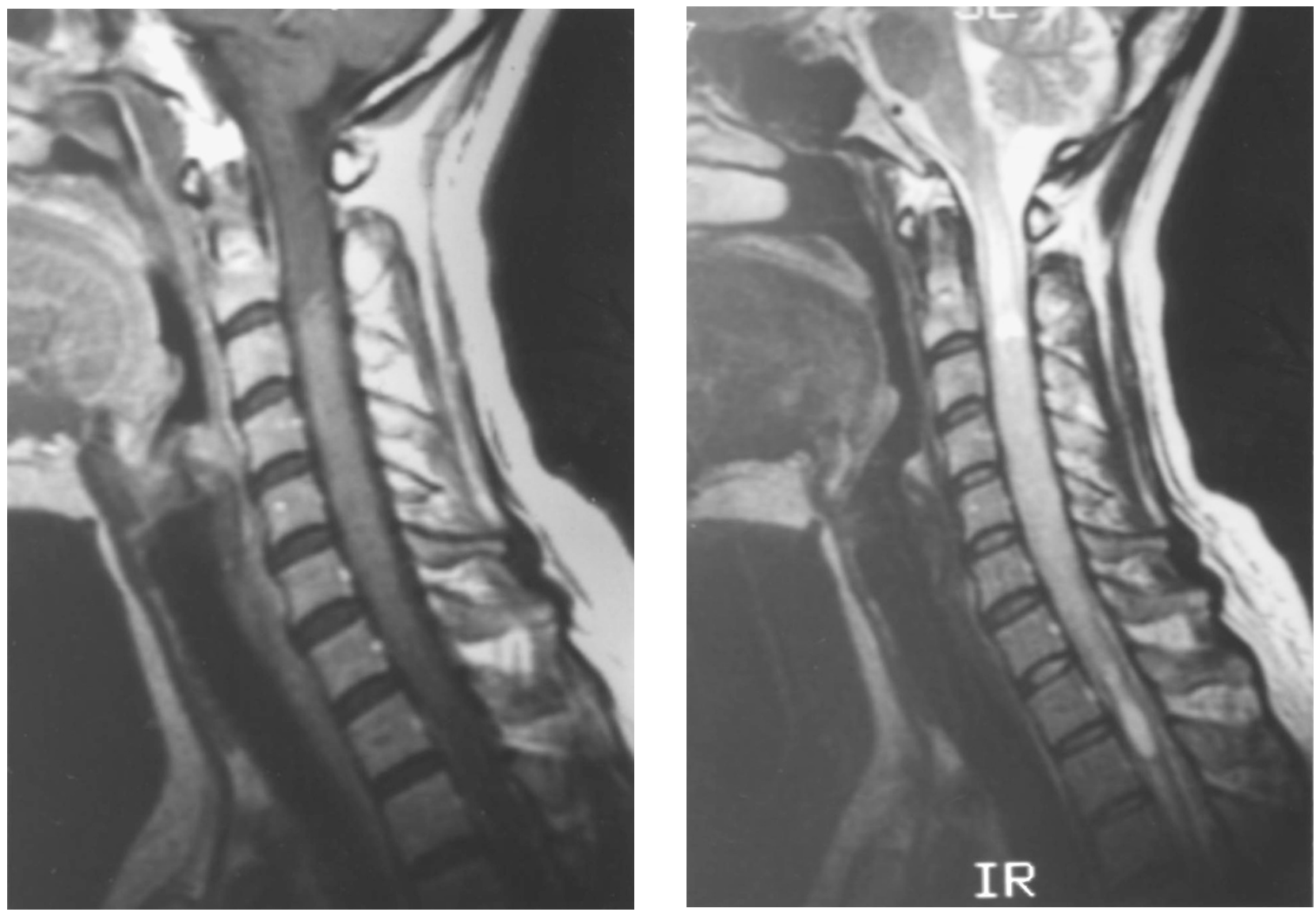

Figure 3 Case, 43-year-old, female. MR image (left: T1W Gd enhanced/right: T2W) demonstrated intramedullary spinal cord tumor at the level from $\mathrm{C} 2$ to Th1

Considering the results of the postoperative neurologic condition in our series, the recovery rate was better with the lumbar lesions than with the cervical and thoracic lesions. Garcia ${ }^{15}$ also found that patients with tumors of the cauda equina had superior neurologic function than those with tumors at other sites, because of the greater concentration of function per unit volume in the spinal cord than in the cauda equina.

The major aim in treatment for spinal cord tumors is to remove them completely without worsening the neurologic deficit. The ratio of total tumor removal is $0 \%-100 \%$ in intramedullary ependymomas $5,9,15,16$ and $0 \%-80 \%$ in cauda equina ependymomas. ${ }^{9,15,17}$ Total tumor removal was achieved in 15 of 26 patients $(57.7 \%)$ in our series. The treatment strategy for intramedullary spinal cord tumors including spinal ependymomas has been controversial. Some advocate minimal surgery and radical radiation, ${ }^{18,19}$ others have reported excellent results with radical resection without radiation. ${ }^{6-8,16}$

Many papers have been published concerning radiotherapy as an adjunctive treatment for intramedullary spinal cord tumors. The necessity of radiotherapy is closely related to the extent of the tumor resection. The effectiveness of postoperative radiotherapy for those patients in whom total tumor re-section was not achieved has been reported. ${ }^{11,12,20-}$ ${ }^{23}$ Mork et $a l^{3}$ have reported that the 10-year survival rate was no different in patients treated with surgery alone than in those with additional radiation therapy. Peschel et $a l^{22}$ have recommended postoperative radiotherapy for the patients who had both total and subtotal resection, while Epstein et $a l^{24}$ have advocated that radiation therapy was not a necessary adjunct following gross total removal of the tumor. In our series, seven patients including three patients who had total tumor removal received postoperative radiation; however, at present it is considered to be unnecessary for patients with complete resection.

McCormick et $a l^{8}{ }^{8}$ Rawlings et $a l^{13}$ and Fischer et $a l^{6}$ have strongly recommended complete removal at the initial procedure because recovery is poor in patients with a more severe preoperative deficit. In addition, local recurrence rates are low and survival rates are excellent in patients with total resection. ${ }^{5,16,20}$ It is possible to remove a high percentage of intramedullary spinal cord tumors with microsurgical techniques ${ }^{4,6}$ without worsening the neurologic condition. 

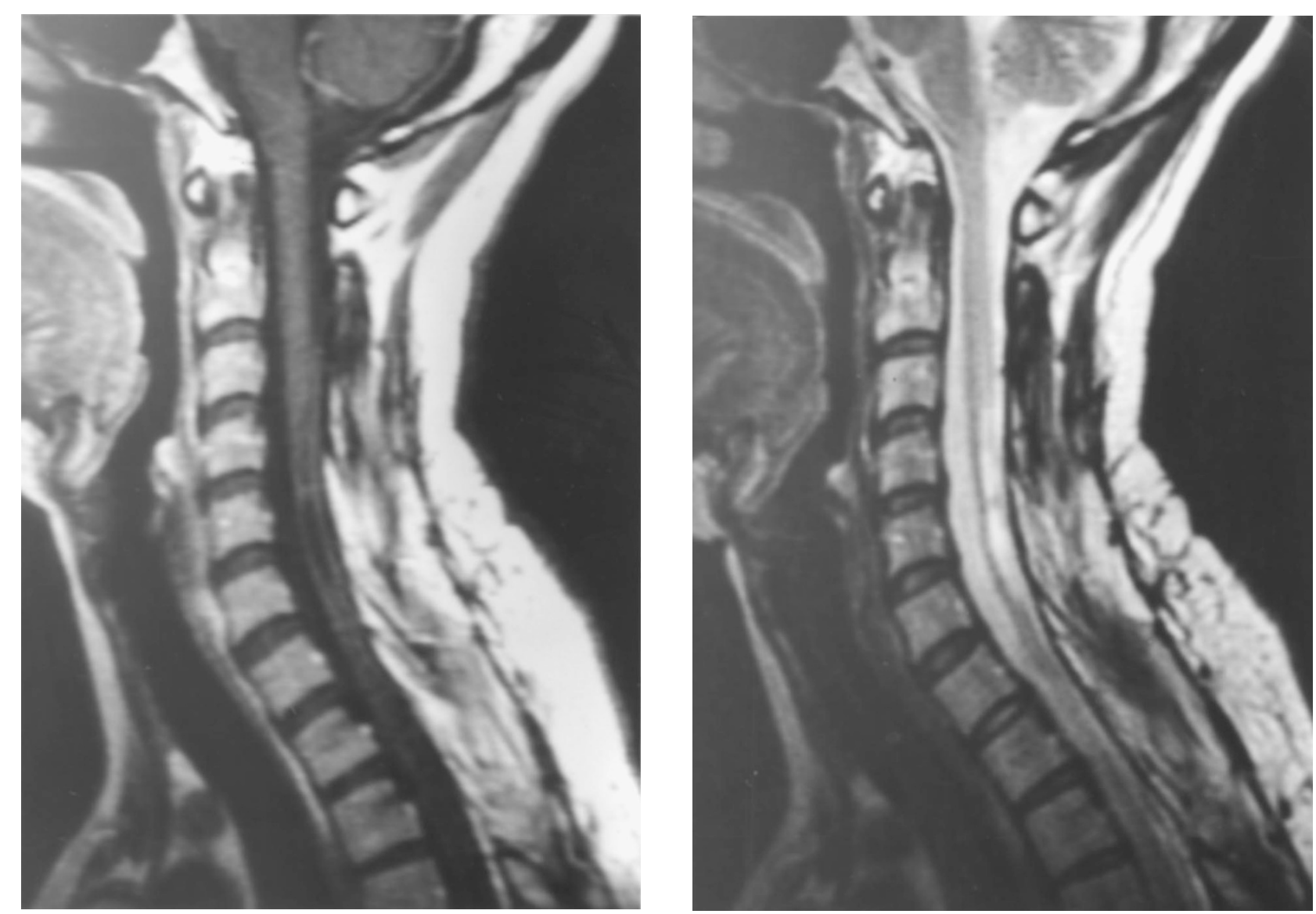

Figure 4 Post operative MR image (left: T1W Gd enhanced/right: T2W) demonstrated no recurrence from the tumors

The literature supports the use of microsurgical techniques to remove intramedullary tumors. ${ }^{4-6,8}$ Greenwood $^{16}$ has detailed the microsurgical approach to intramedullary tumors. However, very few papers have discussed the difference in the clinical results between patients in whom microsurgical techniques and spinal cord monitoring were used intraoperatively and patients in whom they were not used. The use of microsurgical techniques with the addition of spinal cord monitoring has contributed to the improvement of the neurologic recovery rate as McCormick described, ${ }^{8}$ although Cooper et $a l^{7}$ have reported that operative results showed no difference in monitored and nonmonitored patients. We focused on the difference in the clinical results between these two groups. In this study, comparing those patients in whom tumor removal was performed utilizing microsurgical techniques and spinal cord monitoring to those in whom spinal cord monitoring was not used, the neurologic recovery rate was statistically better in the former group. Both microsurgical techniques and spinal cord monitoring are indispensable to achieve the total removal of an ependymoma and to obtain a satisfactory neurologic recovery.

\section{References}

1 Fokes EC, Earle KM. Ependymomas: Clinical and pathological aspects. J Neurosurg 1969; 30: 585-594.

2 Minauf M, Jellinger K, Grunert V. Zur Haufigkeit und Morphologie suptratentorieller Ependymome im Erwachsenenalter. Acta Neurochir 1970; 22: 181-193.

3 Mork SJ, Loken AC. Ependymoma. A follow-up study of 101 cases. Cancer 1977; 40: 907 -915.

4 Garrido E, Stein BM. Microsurgical removal of intramedullary spinal cord tumors. Surg Neurol 1977; 7: 215-219.

5 Malis LI. Intramedullary spinal cord tumors. Clin Neurosurg 1978; 25: $512-539$.

6 Fischer G, Mansuy L. Total removal of intramedullary ependymomas: Follow-up study of 16 cases. Surg Neurol 1980; 14: $243-249$.

7 Cooper PR, Epstein F. Radical resection of intramedullary spinal cord tumors in adults. Recent experience in 29 patients. $J$ Neurosurg 1985; 63: $492-499$.

8 McCormick PC. Spinal ependymoma. Neurosurg Quarterly 1993; 3: $178-191$.

9 Barone BM, Elvdge AR. Ependymomas. A clinical study. $J$ Neurosurg 1970; 33: $428-438$.

10 Woltman HW et al. Intramedullary tumors of spinal cord and gliomas of intradural portion of filum terminale. Fate of patients who have these tumors. Arch Neurol Psychiat 1951; 65: 387-393.

11 Whitaker SJ et al. Postoperative radiotherapy in the management of spinal cord ependymoma. J Neurosurg 1991; 74: 720-728. 
12 Waldron JN et al. Spinal cord ependymomas: A retrospective analysis of 59 cases. Int J Radiation Oncology Biol Phys 1993; 27: $223-229$.

13 Rawlings III CE, Giangaspero F, Burger PC. Ependymomas: A clinicopathologic study. Surg Neurol 1988; 29: $271-281$.

14 Marks JE, Adler SJ. A comparative study of ependymomas by site of origin. Int J Radiat Oncol Biol Phys 1982; 8: 37-43.

15 Garcia DM. Primary spinal cord tumors treated with surgery and post-operative irradiation. Int J Radiat Oncol Biol Phys 1985; 9: $1121-1124$

16 Greenwood J. Intramedullary tumors of the spinal cord. A follow-up study after total surgical removal. J Neurosurg 1963; 20: $665-668$

17 Fearnside MR, Adams CBT. Tumors of the cauda equina. $J$ Neurol. Neurosurg Psych 1978; 41: 24-31.

18 Scott M. Infiltrating ependymomas of the cauda equina. $J$ Neurosurg 1974; 41: $446-448$.
19 Kopelson G, Linggood RM, Kleinman GM. Management of intramedullary spinal cord tumors. Radiology 1980; 135: 473 479

20 Guidett B, Fortuna A. Surgical treatment of intramedullary hemangioblastoma of the spinal cord; report of six cases. $J$ Neurosurg 1967; 27: 530-540.

21 Schwade JG, Wara WM, Sheline GE. Management of primary spinal cord tumors. Int J Radiat Oncol Biol Phys 1978; 4: $389-$ 393.

22 Peschel RE et al. Ependymomas of the spinal cord. Int $J$ Radiat Oncol Biol Phys 1983; 9: 1093 - 1096.

23 Ross DA, McKeever PE, Sandler HM. Myxopapillary ependymoma. Results of nucleolar organizing region staining. Cancer 1993; 71: 3114-3118.

24 Epstein FJ, Farmer JP, Freed D. Adult intramedullary spinal cord ependymomas: the result of surgery in 38 patients. $J$ Neurosurg 1993; 79: 204-209. 